Ferrata Storti Foundation

\title{
Immune reconstitution and associated infections following axicabtagene ciloleucel in relapsed or refractory large B-cell lymphoma
}

Haematologica 2021

Volume 106(4):978-986

\section{Correspondence: \\ FREDERICK L. LOCKE \\ frederick.locke@moffitt.org \\ MICHAEL D. JAIN \\ michael.jain@moffitt.org}

Received: September 21, 2019.

Accepted: April 2, 2020.

Pre-published: April 23, 2020.

https://doi.org/10.3324/haematol.2019.238634

(C)2021 Ferrata Storti Foundation

Material published in Haematologica is covered by copyright. All rights are reserved to the Ferrata Storti Foundation. Use of published material is allowed under the following terms and conditions:

https://creativecommons.org/licenses/by-nc/4.0/legalcode. Copies of published material are allowed for personal or internal use. Sharing published material for non-commercial purposes is subject to the following conditions:

https://creativecommons.org/licenses/by-nc/4.0/legalcode, sect. 3. Reproducing and sharing published material for commercial purposes is not allowed without permission in writing from the publisher.

\author{
Jennifer M. Logue,${ }^{1,2^{*}}$ Elisa Zucchetti, ${ }^{3 *}$ Christina A. Bachmeier, ${ }^{1}$ \\ Gabriel S. Krivenko, ${ }^{1}$ Victoria Larson, ${ }^{2}$ Daniel Ninh, ${ }^{1}$ Giovanni Grillo, ${ }^{3}$ \\ Biwei Cao, ${ }^{4}$ Jongphil Kim, ${ }^{4}$ Julio C. Chavez, ${ }^{2,5}$ Aliyah Baluch, ${ }^{2,6}$ \\ Farhad Khimani,, ${ }^{1,2}$ Aleksandr Lazaryan,, ${ }^{1,2}$ Taiga Nishihori,, ${ }^{1,2}$ Hien D. Liu, 1,2 \\ Javier Pinilla-lbarz, ${ }^{2,5}$ Bijal D. Shah, ${ }^{2,5}$ Rawan Faramand, ${ }^{1,2}$ Anna E. Coghill, ${ }^{7}$ \\ Marco L. Davila, ${ }^{1,2}$ Bhagirathbhai R. Dholaria, ${ }^{1,8}$ Michael D. Jain ${ }^{1,2 \#}$ and \\ Frederick L. Locke ${ }^{1,2 \#}$
}

${ }^{1}$ Department of Blood and Marrow Transplant and Cellular Immunotherapy, H. Lee Moffitt Cancer Center and Research Institute, Tampa, FL, USA; ${ }^{2}$ Morsani College of Medicine, University of South Florida, Tampa, FL, USA; ${ }^{3}$ Divisione di Ematologia, Centro Trapianti di Midollo, ASST Grande Ospedale Metropolitano Niguarda, Milan, Italy; ${ }^{4}$ Department of Biostatistics and Bioinformatics, H. Lee Moffitt Cancer Center and Research Institute, Tampa, FL, USA; ${ }^{5}$ Department of Malignant Hematology, H. Lee Moffitt Cancer Center and Research Institute, Tampa, FL, USA; ${ }^{6}$ Department of Infectious Diseases, H. Lee Moffitt Cancer Center and Research Institute, Tampa, FL, USA; ${ }^{7}$ Cancer Epidemiology Program, H. Lee Moffitt Cancer Center and Research Institute, Tampa, FL, USA and ${ }^{8}$ Department of Hematology-Oncology, Vanderbilt University Medical Center, Nashville, TN, USA

*JML and EZ contributed equally as co-first authors.

"MDJ and FLL contributed equally as co-senior authors

\section{ABSTRACT}

C

D19 chimeric antigen receptor T (CAR T)-cell therapy with axicabtagene ciloleucel (axi-cel) for relapsed or refractory (R/R) large B-cell lymphoma (LBCL) may lead to durable remissions, however, prolonged cytopenias and infections may occur. In this single center retrospective study of 85 patients, we characterized immune reconstitution and infections for patients remaining in remission after axi-cel for LBCL. Prolonged cytopenias (those occurring at or after day 30 following infusion) were common with $\geq$ grade 3 neutropenia seen in 21 of $70(30 \%)$ patients at day 30 and persisting in 3 of $31(9.7 \%)$ patients at 1 year. B cells were undetectable in 30 of $34(88.2 \%)$ patients at day 30 , but were detected in 11 of $19(57.9 \%)$ at 1 year. Median immunoglobulin G levels levels reached a nadir at day 180. By contrast, CD4 T cells decreased from baseline and were persistently low with a median CD4 count of 155 cells/ $\mu \mathrm{L}$ at 1 year after axi-cel ( $\mathrm{n}=19$, range: $33-269)$. In total, 23 of $85(27.1 \%)$ patients received intravenous immunoglobulins after axi-cel, and 34 of $85(40 \%)$ received granulocyte-colony stimulating factor. Infections in the first 30 days occurred in 31 of $85(36.5 \%)$ patients, of which 11 of $85(12.9 \%)$ required intravenous antibiotics or hospitalization ("severe") and were associated with cytokine release syndrome, neurotoxicity, tocilizumab use, corticosteroid use, and bridging therapy on univariate analyses. After day 30, seven severe infections occurred, with no late deaths due to infection. Prolonged cytopenias are common following axi-cel therapy for LBCL and typically recover with time. Most patients experience profound and prolonged CD4 T-cell immunosuppression without severe infection.

\section{Introduction}

Axicabtagene ciloleucel (axi-cel) can lead to long term disease control for patients with R/R LBCL, including diffuse (DLBCL), primary mediastinal (PMBCL), and transformed follicular lymphoma (tFL). In the pivotal ZUMA-1 trial, axi-cel led to a best objective response rate (ORR) of $82 \%$ and complete response (CR) rate of $54 \%$, with 2 -year follow-up data reporting durable responses and median overall 
survival not reached. ${ }^{1,2}$ Major acute side effects of chimeric antigen receptor

$\mathrm{T}$ (CAR T)-cell therapy include cytokine release syndrome (CRS) and neurologic toxicities, which are treated with anti-IL- 6 receptor blockade and/or corticosteroids.

In the ZUMA-1 trial, grade 3 or higher cytopenias were common in the first 30 days following CAR T-cell therapy, and this is typically attributed to fludarabine and cyclophosphamide given for lymphodepletion prior to CAR T-cell infusion., However, cytopenias may persist, and at 3 months or later, $17 \%$ of ZUMA-1 patients experienced one or more grade 3 or higher cytopenia, including $11 \%$ with neutropenia, $7 \%$ with thrombocytopenia, and $3 \%$ with anemia. ${ }^{1}$ Late cytopenias were seen without evidence of marrow dysplasia or relapse. In addition, B-cell aplasia occurred due to on-target elimination of CD19-expressing normal B cells, with resultant hypogammaglobulinemia, and use of intravenous immunoglobulins (IVIG) in $31 \%$. Overall, $28 \%$ of patients had $\geq$ grade 3 infections on the ZUMA-1 trial.

The presence of early and late cytopenias, corticosteroid treatment for CRS and neurotoxicity, and reconstitution of $\mathrm{B}$ and $\mathrm{T}$ lymphocytes after CAR $\mathrm{T}$ therapy may put patients at risk of infection. This study aimed to characterize immune reconstitution after axi-cel therapy and identify early and late infections in patients with R/R LBCL receiving treatment with axi-cel. We examined cytopenias, lymphocyte reconstitution, and infection data up to 1 year following infusion of axi-cel.

\section{Methods}

\section{Patients and data collection}

We retrospectively reviewed data from the medical records of patients with R/R LBCL who were treated with axi-cel at the Moffitt Cancer Center between February 1, 2016, and February 28, 2019. This study was approved by the Institutional Review Board. Data extracted from the electronic medical record included patient demographics, prior therapies, baseline disease status, CAR T-cell product, dates of treatment and disease progression or last follow-up, occurrence and grade of CRS and neurotoxicity, complete blood counts ( $\mathrm{CBC}$ ), immunoglobulin levels, infection data, pathology reports, and drug administration. B-, T- and natural killer-lymphocyte subsets were quantified from fresh peripheral blood samples using a validated flow cytometry panel in the clinical lab. All data was censored at date of progression, development of a new malignancy requiring systemic treatment, death, or last follow-up, in order to understand the effect of axi-cel therapy independent of disease progression. Immunoglobulin levels were censored after a patient was treated with IVIG. Adverse events were graded per the Common Terminology Criteria for Adverse Events (CTCAE) v4.03. CRS was scored based on modified Lee grading system. ${ }^{5}$ Neurologic toxicity was scored based on CARrelated encephalopathy syndrome/CAR T toxity (CRES/CARTOX) grading system or individual terms for CTCAE neurotoxicity. ${ }^{6}$ Disease status at apheresis was defined as: primary refractory, never achieving end of treatment CR; refractory, not primary refractory and no response to the most recent therapy; relapsed, responded to most recent therapy and progressed. Bridging therapy was defined as any lymphoma-specific therapy administered after leukapheresis and before conditioning chemotherapy. Cyclophosphamide and fludarabine conditioning followed by axicel infusion were performed as in ZUMA-1. Prophylaxis policies were adapted from our institution's autologous stem cell trans- plant procedures. Our institutional standard for antimicrobial prophylaxis includes starting antibacterial prophylaxis with a fluoroquinolone and antifungal prophylaxis with fluconazole on the morning of axi-cel infusion or earlier if the patient is neutropenic, with discontinuation in afebrile patients free of infection after neutrophil recovery. The standard empiric treatment for neutropenic fever included cefepime or piperacillin/tazobactam. Pneumocystis jiroveci pneumonia (PJP) prophylaxis is started on day 30 following axi-cel infusion and included sulfamethoxazole-trimethoprim in patients without cytopenias, with inhaled pentamidine, dapsone or atovaquone prophylaxis used in cytopenic patients. Duration of PJP prophylaxis was physician dependent and was typically provided for 6-12 months, or until recovery of CD4 count over 200 cells/ $\mu \mathrm{L}$. Varicella zoster virus (VZV) reactivation prophylaxis is provided for a minimum of 12 months, typically using acyclovir. Herpes simplex virus (HSV) 1/2 immunoglobulin G (IgG) testing was by ARUP laboratories (Salt Lake City, UT).

\section{Definition of infection}

An episode of infection was defined as a viral, bacterial, or fungal finding based on microbiological data or a clinical syndrome, which was found based on retrospective chart review. Severe infection was defined as an infection which required intravenous (IV) antibiotics or hospitalization. Standard follow-up at our institution includes clinic appointments on days 30, 90, 180, 270, and 360. Patients were expected to call if they developed any infectious symptoms in the interim.

\section{Statistical analysis}

Patient characteristics were summarized using descriptive statistics including median and range for continuous measures and proportions and frequencies for categorical measures. Chi-square test or Fisher exact test was used to explore the association between categorical variables. Dunnett's test was used to make pairwise comparisons with Day 0, and Kruskal-Wallis test has been used to compare total white blood cells (WBC), neutrophils (N), CD3 positive cells, CD56 positive cells, CD8 positive cells, CD4 positive cells, CD19 positive cells, and IgG levels up to 1 year following axi-cel. When comparing characteristics to infection/severe infection, the associations between categorical variables were evaluated using $\chi^{2}$ tests or Fisher's exact tests when the expected frequency was less than 5 . In addition, infection incidence per 1,000 person-days at day 30, 90, 180, 270, and 360 was computed to incorporate the multiple infections per patient. The cumulative incidence of first infection was estimated by competing risk approach, where death or progression were considered as competing events. The association between continuous variables and infection were assessed using Wilcoxon tests. Categorical variable levels for overall survival (OS) were compared using the Log-rank test. Kaplan-Meier curves were used to show progression free survival (PFS) and OS for all patients in the study. Statistical significance was defined as two-sided $P$-value of $<0.05$.

\section{Results}

The majority of acute toxicities are known to resolve before day 30 after CAR T, which also is the time of first disease assessment. ' Therefore, we separately analyzed immune reconstitution and infections prior to day 30 and after day 30 after axi-cel infusion. Further, in order to focus on survivorship we censored patients from the analysis at the time of lymphoma relapse. Of 85 infused patients, we censored 11 for analysis beyond day 30 due to early progressive disease or death, and an additional 4 patients 
Table 1. Patient characteristics and clinical outcomes.

Axi-cel

Standard of Care

Clinical trial

Age, years

Median (range)

$\geq 65$

Sex

Female

ECOG at apheresis

0

1

2

3

Disease stage at apheresis

I or II

III or IV

International Prognostic Index score at apheresis

$0-2$
$3-5$
Presence of bulky disease

Presence of bulky disease

Disease type

Diffuse large B-cell lymphoma

Transformed follicular lymphoma

Primary mediastinal B-cell lymphoma

Prior lines of therapies

Median (range)

1

2

$\geq 3$

Prior autologous stem cell transplant

Prior allogeneic stem cell transplant

Bridging therapy

Disease status

Primary refractory

Refractory

Relapsed

Cytokine release syndrome maximum grade

0

1

$3-4$

CAR T-cell related encephalopathy syndrome maximum grade

0

$1-2$

$3-4$

Treatment for toxicity

\section{Steroid use}

Tocilizumab use

30-day response

$\begin{array}{lc}\text { ORR } & 68(80.0 \%) \\ \text { CR } & 41(48.2 \%) \\ \text { PR } & 27(31.8 \%) \\ \text { SD } & 9(10.6 \%) \\ \text { PD } & 7(8 \cdot 2 \%) \\ \text { No response assessment } & 1(1.2 \%)\end{array}$

No response assessment

Patient baseline characteristics prior to axi-cel and clinical outcomes following axi-cel infusion for patients analyzed prior to and after day 30. axi-cel: axicabtagene ciloleucel; IQR: interquartile range; ECOG: Eastern Cooperative Oncology Group performance status; Bulky disease: any mass diameter greater than $10 \mathrm{~cm}$; ORR: overall response rate (calculated as complete response [CR] + partial response [PR]); SD: stable disease; PD: progressive disease; CAR T: chimeric antigen receptor T . Disease status at apheresis was defined as: primary refractory, never achieving end of treatment $\mathrm{CR}$; refractory, not primary refractory and no response to the most recent therapy; relapsed, responded to most recent therapy and progressed.

$48(68.6 \%)$

$22(31.4 \%)$

$63(28-79)$

$29(41.4 \%)$

27 (38.6\%)

$26(37 \cdot 1 \%)$

$34(48.6 \%)$

9 (12.9\%)

$1(1.4 \%)$

17 (24.3\%)

53 (75.7\%)

$28(32.9 \%)$

27 (38.6\%)

$43(61.4 \%)$

$20(23.5 \%)$

13 (18.6\%)

$56(65.9 \%)$

$47(67 \cdot 1 \%)$

$20(28.6 \%)$

$3(4.3 \%)$

3 (3.5\%)

3 (1-7)

$3(4.3 \%)$

25 (35.7\%)

42 (60.0\%)

17 (24.3\%)

$2(2.9 \%)$

$36(51.4 \%)$

$22(31.4 \%)$

25 (35.7\%)

$23(32.9 \%)$

$31(36.5 \%)$

$4(5.7 \%)$

$34(48.6 \%)$

29 (41.4\%)

$3(4.3 \%)$

$35(41.2 \%)$

25 (35.7\%)

28 (40.0\%)

$17(24.3 \%)$

27 (38.6\%)

28 (40.0\%)

64 (91.4\%)

38 (54.3\%)

$26(37.1 \%)$

$6(8.6 \%)$

$0(0.0 \%)$

$0(0.0 \%)$

$\begin{array}{ll}39(45.9 \%) & 27(38.6 \%) \\ 39(45.9 \%) & 28(40.0 \%)\end{array}$

(80.0\%)

$31(36.5 \%)$

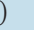


Received axi-cel infusion for LBCL and analyzed for immune reconstitution and infection before day $30(\mathrm{n}=85)$

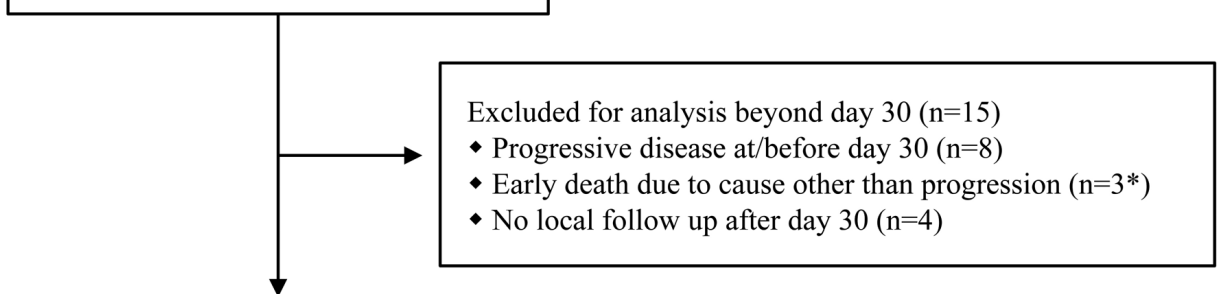

Analyzed for immune reconstitution and infection beyond day $30(\mathrm{n}=70)$
Figure 1. Cohort description. *Early deaths after chimeric antigen receptor T (CAR T) therapy included death due to fusarium infection in the central nervous system at day 39 , hemophagocytic lymphohistiocytosis at day 38 , and candidemia at day $40 . .^{19}$

Table 2. Cytopenias following axicabtagene ciloleucel (axi-cel).

\begin{tabular}{|c|c|c|c|c|c|c|}
\hline & Apheresis & Day 30 & Day 90 & Day 180 & Day 270 & Day 360 \\
\hline Grade 3-4 Leukopenia & $3.5 \%(3 / 85)$ & $28.6 \%(20 / 70)$ & $10.7 \%(6 / 56)$ & $16.7 \%(7 / 42)$ & $9.4 \%(3 / 32)$ & $3.2 \%(1 / 31)$ \\
\hline Any Grade Leukopenia & $23.5 \%(20 / 85)$ & $85.7 \%(60 / 70)$ & $64.3 \%(36 / 56)$ & $64.3 \%(27 / 42)$ & $56.3 \%(18 / 32)$ & $51.6 \%(16 / 31)$ \\
\hline Grade 3-4 Neutropenia & $3.5 \%(3 / 85)$ & $30.0 \%(21 / 70)$ & $12.5 \%(7 / 56)$ & $11.9 \%(5 / 42)$ & $9.4 \%(3 / 32)$ & $9.7 \%(3 / 31)$ \\
\hline Any Grade Neutropenia & $14.1 \%(12 / 85)$ & $71.4 \%(50 / 70)$ & $37.5 \%(21 / 56)$ & $42.9 \%(18 / 42)$ & $37.5 \%(12 / 32)$ & $25.8 \%(8 / 31)$ \\
\hline Grade 3-4 Anemia & $7.1 \%(6 / 85)$ & $7.1 \%(5 / 70)$ & $3.6 \%(2 / 56)$ & $7.1 \%(3 / 42)$ & $3.1 \%(1 / 32)$ & $3.2 \%(1 / 31)$ \\
\hline Any Grade Anemia & $57.6 \%(49 / 85)$ & $87.1 \%(61 / 70)$ & $44.6 \%(25 / 56)$ & $31.0 \%(13 / 42)$ & $31.3 \%(10 / 32)$ & $22.6 \%(7 / 31)$ \\
\hline Grade 3-4 Thrombocytopenia & $5.9 \%(5 / 85)$ & $25.7 \%(18 / 70)$ & $5.4 \%(3 / 56)$ & $4.8 \%(2 / 42)$ & $3.1 \%(1 / 32)$ & $3.2 \%(1 / 31)$ \\
\hline Any Grade Thrombocytopenia & $28.2 \%(24 / 85)$ & $80 \%(56 / 70)$ & $51.8 \%(29 / 56)$ & $45.2 \%(19 / 42)$ & $43.8 \%(14 / 32)$ & $38.7 \%(12 / 31)$ \\
\hline Any Grade 3-4 Cytopenia & $14.1 \%(12 / 85)$ & $44.3 \%(31 / 70)$ & $14.3 \%(8 / 56)$ & $19.0 \%(8 / 42)$ & $9.4 \%(3 / 32)$ & $9.7 \%(3 / 31)$ \\
\hline Any Grade Cytopenia & $72.9 \%(62 / 85)$ & $100.0 \%(70 / 70)$ & $83.9 \%(47 / 56)$ & $81.0 \%(34 / 42)$ & $71.9 \%(23 / 32)$ & $67.7 \%(21 / 31)$ \\
\hline
\end{tabular}

Clinical complete blood count and differential results were graded using clinical trial criteria (CTCAE v4.03). Grade $3-4$ leukopenia $=$ white blood cell count $(\mathrm{WBC})<2,000 / \mathrm{mm}^{3}$, grade 3-4 neutropenia = neutrophil $(\mathrm{N})$ count $<1,000 / \mathrm{mm}^{3}$; grade $3-4$ anemia $=$ hemoglobin $(\mathrm{Hb})<8 \mathrm{~g} / \mathrm{dL}$; grade $3-4$ thrombocytopenia $=$ platelet $(\mathrm{PLT})$ count $<50,000 / \mathrm{mm}^{3}$. For any grade cytopenia, patients had at least grade 1 leukopenia, neutropenia, anemia, or thrombocytopenia, defined as WBC $<4,000 / \mathrm{mm}^{3}, \mathrm{~N}<1,800 / \mathrm{mm}^{3}, \mathrm{Hb}<11.4 \mathrm{~g} / \mathrm{dl}$, and PLT $<143,000 / \mathrm{mm}^{3}$, based on the lower limits of normal set by the clinical laboratory at our institution. Information is shown as \% (\#patients/total number of non-relapsing patients with follow-up at that time point)

because they followed up outside of our institution (Figure 1). Median follow-up from time of axi-cel infusion was 12.8 months (range: 0.8-42.4). The median time from apheresis to axi-cel infusion was 27 days (range: 20-42). Patient characteristics are shown in Table 1. Severe (grade 3 or higher) CRS occurred in $9.4 \%$ of patients, and severe neurotoxicity occurred in $30.6 \%$. Median duration of corticosteroid treatment for toxicity was 7 days (range: 1-62). ORR at day 30 was $80 \%$, and CR at day 30 was $48.2 \%$. Kaplan-Meier curves of PFS and OS are shown in the Online Supplementary Figure S1.

In order to describe cytopenias after axi-cel, we graded cytopenias based on the clinical $\mathrm{CBC}$ and differential using clinical trial criteria (CTCAE v4.03; Table 2). At baseline (time of apheresis) prior to axi-cel, $14.1 \%$ of patients had grade 3 or 4 cytopenias. At day 30 after axicel, grade 3 or 4 leukopenia, neutropenia, or thrombocytopenia was found in $28.6 \%, 30 \%$, and $25.7 \%$ of patients, respectively, for a cumulative grade 3 or 4 cytopenia rate of $44.3 \%$. All patients at day 30 had at least one grade 1 cytopenia. Most patients recovered counts over subsequent months, although a proportion of patients ( 3 of 31 ; $9.7 \%$ ) still had neutrophil counts below $1,000 / \mathrm{mL}$ at 1 year after axi-cel infusion.

Cellular and humoral reconstitution was evaluated over time using the clinical $\mathrm{CBC}$ and a flow cytometry panel that quantifies B-, T-, and NK-cell subsets (Figure 2).
Median leukocyte and neutrophil counts decreased after axi-cel therapy and remained significantly below baseline levels at 1 year after therapy. Similarly, peripheral blood CD4 T cells were low at a median of 220 cells/ $\mu \mathrm{L}$ (range: 34-1,720) prior to axi-cel and remained persistently low after axi-cel, with a median CD4 count of 155 cells/ $\mu \mathrm{L}$ (range: 33-269) at 1 year. Multiple comparison testing and means are provided in the Online Supplementary Table S1. CD19 positive B cells were detectable in 28 of 58 (48.3\%) patients at baseline prior to axi-cel and in 4 of $34(11.8 \%)$ patients at day 30 . The proportion of patients with detectable levels at day 90,180 , and 360 , were $22.6 \%$ (7 of 31 ), $46.2 \%$ (12 of 26), and $57.9 \%$ (11 of 19), respectively; $\mathrm{B}$ cells had significantly increased from baseline by 1 year $(P=0.05)$. At baseline 9 of $58(15.5 \%)$ patients had IgG $<300 \mathrm{mg} / \mathrm{dL}, 16$ of $58(27.6 \%)$ patients had IgG $<400$ $\mathrm{mg} / \mathrm{dL}$, and 27 of $58(46.6 \%)$ patients had IgG $<500$ $\mathrm{mg} / \mathrm{dL}$. IgG levels decreased after axi-cel infusion with a nadir at 6 months. By 1 year, excluding patients censored for receipt of IVIG, 9 of 17 (52.9\%) had IgG levels above $400 \mathrm{mg} / \mathrm{dL}$ and 7 of $17(41.2 \%)$ patients had IgG levels above $500 \mathrm{mg} / \mathrm{dL}$. Of the patients with $\mathrm{IgG} \geq 500 \mathrm{mg} / \mathrm{dL}$ at baseline, new hypogammaglobulinemia occurred in 10 of $17(58.8 \%)$ that were measured at day 30 and 20 of 27 $(74.1 \%)$ in total at any time. We also followed quantitative titers of HSV antibodies in 17 patients (Online Supplementary Figure S2). While quantitative levels 

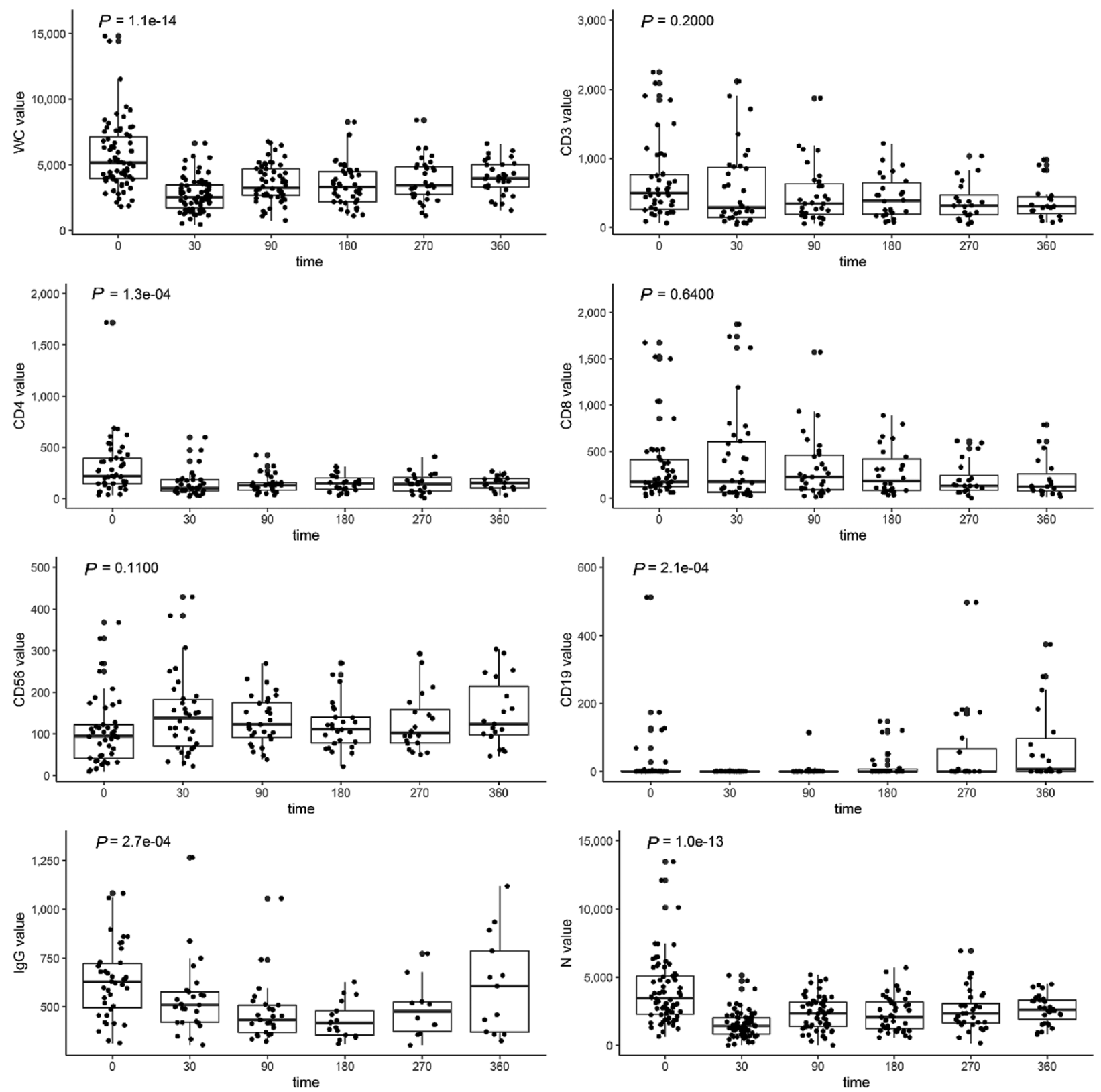

Figure 2. Cellular and humoral immune reconstitution after axicabtagene ciloleucel (axi-cel). Box and whisker plots demonstrating cellular and immune reconstitution following treatment with axi-cel. White blood cells $(\mathrm{WC})$ and neutrophils $(\mathrm{N})$ were measured by complete blood counts with differential at baseline ( $\mathrm{n}=85$ ), day $30(n=70)$, day $90(n=56)$, day $180(n=42)$, day $270(n=32)$, and day $360(n=31)$. CD3 T cells, CD4 T cells, CD8 T cells, CD56 natural killer cells, and CD19 B cells were measured by flow cytometry at baseline $(n=58)$ and at day $30(n=34), 90(n=31), 180(n=26), 270(n=20)$ and 360 ( $n=19)$. Also shown are serum mmunoglobulin G (IgG) levels at baseline $(n=58)$ and at day $30(n=34), 90(n=31), 180(n=19), 270(n=17)$ and $360(n=17)$ after treatment with axi-cel. Boxes demonstrate first quartile, median and third quartile values. Whiskers show the data ranges. Dots represent individual patients. $P$-values are calculated by the Kruskal-Wallis test to assess significant differences in the indicated cell type after axi-cel infusion.

decreased, no patient fell below the minimum titer for a positive test during the follow-up period. In total, 23 of 85 $(27.1 \%)$ patients required IVIG after axi-cel. 34 of 85 $(40.0 \%)$ patients required G-CSF due to severe neutropenia.

A proportion of patients had bone marrow biopsies done pre-treatment and after axi-cel therapy (Table 3). The median pre-treatment marrow occurred at day -179 (range: -1551 to -7 ). Before axi-cel, $21.5 \%$ of patients had a hypocellular marrow, $60 \%$ of patients had a normocellular marrow, no patients had dysplasia, $10.8 \%$ had mild to moderate fibrosis, and $16.9 \%$ had a clonal population.
Of marrows with a clonal population, four showed DLBCL, three follicular lymphoma (FL), one marginal zone lymphoma (MZL), one chronic lymphocytic leukemia (CLL), one monoclonal plasma cell proliferation in the setting of monoclonal gammopathy of undetermined significance, and one had monoclonal $\mathrm{CD}^{+} \mathrm{B}$ cells. Of these patients, seven went on to have repeat bone marrow biopsies after treatment with axi-cel, and no patients showed persistence of the clonal population at the time of repeat biopsy. Within the first 100 days after axi-cel, 16 patients had bone marrow biopsies done in the absence of lymphoma progression, occurring at median day 31 


\begin{tabular}{lccc} 
& Pre-axi-cel & Day 1-100 & Day 101-360 \\
Median Day (range) & $-179(-1551$ to -7$)$ & $31(6$ to 100$)$ & $202(102$ to 289$)$ \\
Hypocellular marrow & $21.5 \%(14 / 65)$ & $87.5 \%(14 / 16)$ & $50.0 \%(3 / 6)$ \\
\hline Normocellular marrow & $60.0 \%(39 / 65)$ & $12.5 \%(2 / 16)$ & $50.0 \%(3 / 6)$ \\
Hypercellular marrow & $18.5 \%(12 / 65)$ & $0.0 \%(0 / 16)$ & $0.0 \%(0 / 6)$ \\
\hline Dysplasia Present & $0.0 \%(0 / 65)$ & $6.3 \%(1 / 16)$ & $33.3 \%(2 / 6)$ \\
Fibrosis Present & $10.8 \%(7 / 65)$ & $31.3 \%(5 / 16)$ & $33.3 \%(2 / 6)$ \\
\hline
\end{tabular}

axi-cel: axicabtagene ciloleucel. Bone marrow cellularity as designated by the clinical pathologist. Clinical criteria account for patient age and are defined as hypocellular with a cell-to-fat ratio of 1:3 or less and hypercellular with a cell-to-fat ratio of 3:1 or greater. ${ }^{20}$

(range: 6-100). Of these, $87.5 \%$ showed hypocellularity, $6.3 \%$ had evidence of dysplasia, and $31.3 \%$ displayed mild fibrosis. Beyond the first 100 days after axi-cel, six bone marrow biopsies were done at median day 202 (range: 102-289). Of these, three were hypocellular and three were normocellular. Dysplasia and fibrosis were observed in four patients, with two patients having both dysplasia and fibrosis on biopsy, one patient with fibrosis only, and one patient with dysplasia only.

In the first 30 days, infection occurred in 31 of 85 patients $(36.5 \%)$. Of these, 12 patients had Clostridium difficile $(14.1 \%)$, and 10 patients had a respiratory virus $(11.8 \%)$ (Figure 3A, Online Supplementary Table S2A). At the time of Clostridium difficile detection, one patient was on fluoroquinolone prophylaxis, while eight were receiving broad spectrum antibiotics for neutropenic fever and three patients were not on any antibiotics. We further classified the infections as severe or non-severe on the basis of whether IV antibiotics were required or if hospitalization was warranted, and 11 patients $(12.9 \%)$ had at total of 13 severe infections in the first 30 days. Two patients experienced two severe infections each. These severe infections included six cases of bacteremia, one cellulitis, one acute cholecystitis, one urinary tract infection, two fungal infections (candidemia and fusariosis) and two viremias (adenovirus and cytomegalovirus). For the case of candidemia, cultures drawn on day 38 post axi-cel eventually became positive for Candida krusei (5 days later on day 43). The patient was on antifungal prophylaxis with fluconazole, and autopsy revealed disseminated candidemia. The case of Fusarium was only discovered upon autopsy; the patient did not have positive fungal cultures and had received antifungal prophylaxis starting with fluconazole, and at the time of death, micafungin. Neither patient who experienced a fungal infection had a prior allogeneic stem cell transplant. Both patients received five prior lines of therapy before axi-cel and were treated with prolonged steroids for neurotoxicity after axi-cel. For the two cases of viremia, both patients received antiviral prophylaxis with acyclovir. The most common organisms were gram positive and the fungal isolates were not susceptible to fluconazole (Online Supplementary Table S3).

Severe infections before day 30 were associated on univariate analyses with severe CRS, severe neurotoxicity, tocilizumab use, steroid use, and bridging therapy $(P=0.007, P=0.007, P=0.03, P=0.004$, and $P=0.02$, respectively) (Figure 3B-C, Online Supplementary Table S4). The presence of infection was associated with a higher baseline total white blood cell (WBC) and neutrophil count $(P=0.03$ and $P=0.02$, respectively) (Online Supplementary Table S5).

After 30 days from time of axi-cel infusion, we identified 32 infections in 31 of 70 (44.3\%) patients (Online
Supplementary Table S2B). Of these, 25 infections were non-severe with 19 cases of upper respiratory viruses, one case of bacterial pneumonia, two cases of bacterial sinusitis, one case of Clostridium difficile, one cellulitis, and one groin abscess, all managed with oral antibiotics. The remaining seven cases were severe, including five cases of pneumonia, one case of sepsis due to methicillin-resistant Staphylococcus aureus bacteremia, and one case of neutropenic fever resolving with IV antibiotics.

In total, infection was a contributor to death in three cases $(3.5 \%)$, all early after axi-cel, including both cases of fungal infection and one case of Streptococcus mitis bacteremia co-occurring with rapid lymphoma progression. All other deaths on this study occurred after lymphoma progression.

On a rate basis, infection incidence decreased from 11.7 incident infections per 1,000 person-days in the first 30 days to 2.3 incident infections per 1,000 person-days between day 31 and day 90, and incidence continued to decrease over time (Figure 4A). However, estimates are affected by the competing risk of lymphoma progression or death (Figure 4B).

We evaluated if cytopenias and/or poor immune reconstitution at day 30 were associated with infection after day 30 (Online Supplementary Figure S3). CD4 and CD8 Tcell counts were not significantly lower at day 30 in patients who would go on to develop infection compared to those who remained infection-free. Similarly, day 30 IgG levels were not lower in patients who went on to develop infection compared to those who did not.

\section{Discussion}

Patients with lymphoma receiving fludarabine and cyclophosphamide followed by CD19 CAR T-cell therapy are at risk of acute and chronic immunosuppression. This is due to tumor-related immunosuppression, residual effects of prior lines of lymphoma therapy, lymphodepletion due to fludarabine and cyclophosphamide, B-cell aplasia with hypogammaglobulinemia, immunosuppressive corticosteroids needed for toxicity treatment, and prolonged cytopenias possibly due to cytokine and/or chemokine effects. ${ }^{8-11}$ Here we have characterized immune reconstitution and associated infections in a retrospective cohort of patients receiving a single CAR T product, axicel, in patients with $\mathrm{LBCL}$.

First, we confirm that cytopenias are common after axicel therapy and may persist for months after therapy. On bone marrow biopsy the typical finding is of a normocellular to hypocellular marrow, although cases of myelodysplastic syndrome have been reported after axi-cel. ${ }^{12}$ Attribution of bone marrow changes to axi-cel is unclear 
A

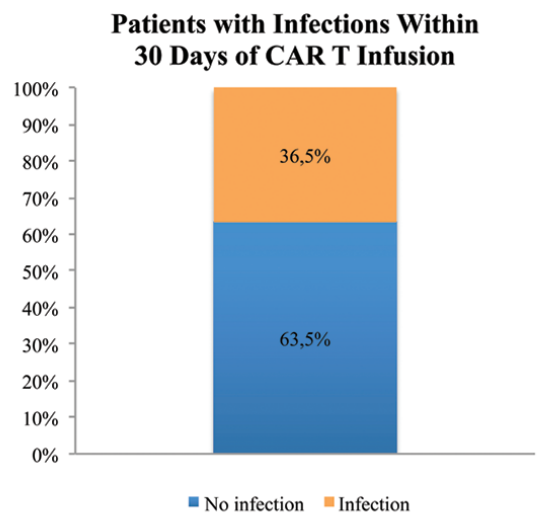

B

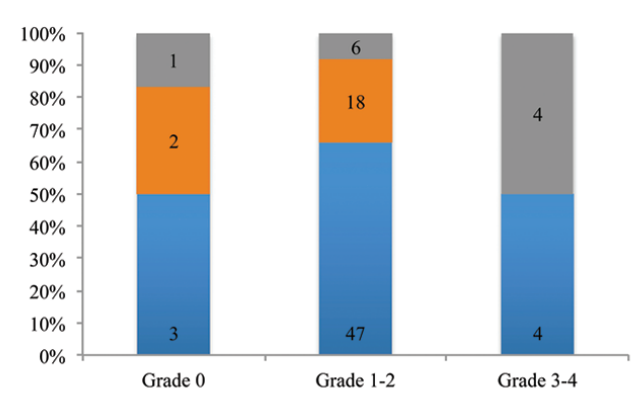

- No infection " Infection not requiring IV antibiotics " Severe infection

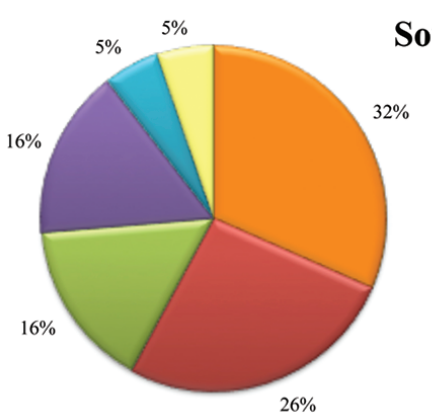

Sources of Infection

Clostridium difficil

"Respiratory virus

অOther

$\varpi_{\text {Bacteremia }}$

Fungal

$\square$ Other Virus
C

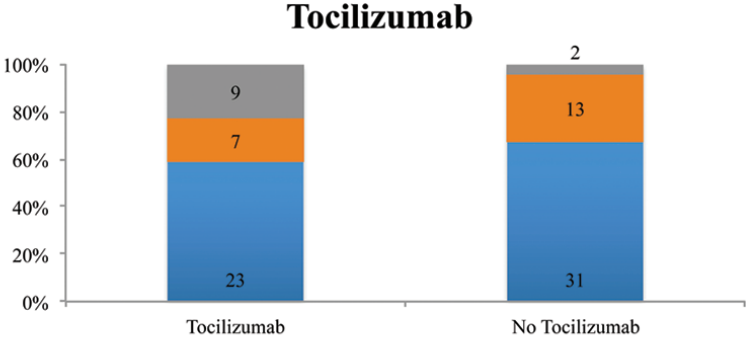

=No infection $\quad$ Infection not requiring IV antibiotics $\quad$ Severe infection

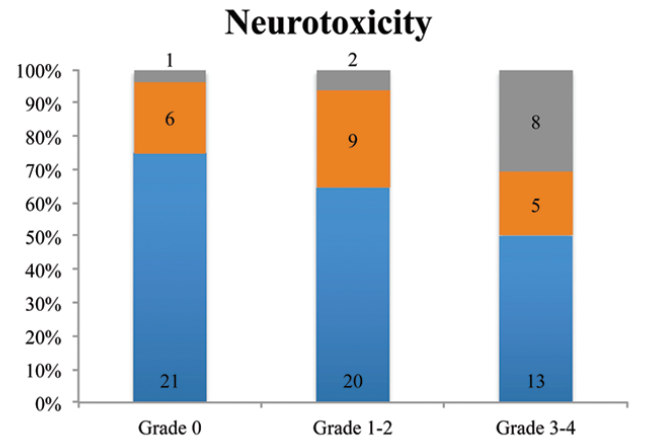

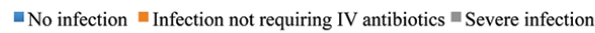

Figure 3. Infections in the first 30 days after axi-cel therapy for aggressive B-cell lymphoma. (A) Rate and type of infection observed in 85 patients in the first 30 days after axi-cel infusion. (B) Infection by grade of cytokine release syndrome (CRS) or neurotoxicity. (C) Non-severe and severe infections in the first 30 days by receipt of anti-IL-6R therapy (tocilizumab) or steroid therapy. CRS: cytokine release syndrome.

given that $65.9 \%$ of the original cohort received at least 3 prior lines of therapy. However a limitation of this study is that there is no standardized schedule for bone marrow biopsies at our institution before or after treatment with axi-cel. Rather, biopsies were done at the discretion of the attending physician with the primary reason for bone marrow biopsy after axi-cel being prolonged cytopenias. Therefore, this data set may not capture all dysplasia or fibrosis events that occur pre- or post-axi-cel therapy. Ultimately, a prospective study including bone marrow biopsies would be helpful.

The mechanism for prolonged cytopenias after CAR T-cell therapy is unclear. Purine analogues such as fludarabine are often associated with prolonged cytopenias, but responding patients generally recover lymphocyte counts and cytopenias within weeks to months after fludarabine and cyclophosphamide, even after six cycles such as is given in CLL. ${ }^{13,14}$ Fried et al. studied 38 pediatric and adult patients after CD19 CAR T therapy for B-cell lymphomas or acute lymphoblastic leukemia (ALL) in the first 2 months after therapy and found a moderate correlation between neutrophil counts and soluble SDF-1. ${ }^{8}$ Our data suggest that although patients may have hypocellular bone marrows and severe cytopenias in the months after axi-cel, supportive care is effective as patients generally recover their counts over subsequent months.

In our cohort, $36.5 \%$ of patients were diagnosed with infections within 30 days after axi-cel, and $44.3 \%$ of patients had infections between days 31 and 360 . Hill and colleagues previously reported on infections following treatment with a CD19/4-1BB/CD3z/EGFRt CAR T-cell therapy for ALL, CLL, and non-Hodgkin lymphoma on clinical trials. ${ }^{3}$ They saw that $23 \%$ of patients had infections within 28 days, and $14 \%$ of patients had infections between days 29 and 90 . Park and colleagues reported on infections occurring after treatment with a 
CD19/CD28/CD3z CAR in R/R B-ALL treated through a phase I trial and found that $42 \%$ of patients had infections during the first 30 days after axi-cel infusion, while 31\% of surviving patients had infections between day 31 and day $180 .{ }^{15}$ Overall, infection rates in our cohort were similar to that reported on these trials, although we characterized most of these infections as non-severe.

In the cohort of lymphoma patients described here, most of the infections in the first 30 days were due to Clostridium difficile colitis or respiratory viruses and were manageable. The rate of Clostridium difficile is likely influenced by our choice of fluoroquinolone prophylaxis for all CAR T patients. Severe infections occurred in $12.9 \%$ of patients, and in $3.5 \%$ of patients, infection was a contributor to death. We find that early severe infections almost always occur in patients that have severe CRS and/or neurotoxicity and receive treatment with tocilizumab, corticosteroids, and/or had bridging therapy. At baseline, total WBC and neutrophil counts were counterintuitively higher in patients with infection compared to those who do not experience infection. Speculatively, patients with higher neutrophil counts at baseline may have adverse lymphoma biology and a greater degree of tumor-related immunosuppression. ${ }^{16,17}$ We also noted that the majority of bacterial isolates in severe infection were gram positive, which may also be related to our use of fluoroquinolone prophylaxis to prevent gram negative infections. The two fungal infections were not susceptible to our prophylactic fluconazole. Clinically, patients who require steroids for toxicity management are at a higher infection risk. There is an overall lack of data to guide prophylaxis policies following treatment with CAR T-cell therapy, and further study is needed to optimize infection prophylaxis and prevent infection in this group.

We also report that CD4 T-cell levels remain persistently low for months after axi-cel. Indeed, we observed that CD 4 counts remain significantly low even at 1 year after treatment. Despite profound and prolonged T-cell immunosuppression, severe infections remain rare. Our low rate of severe infection is in agreement with the smaller study published by Kochenderfer and colleagues, in which only 1 of 7 patients (14.3\%) had a severe infection requiring hospitalization after treatment with CD19 CAR T-cells for LBCL..$^{10}$ Longer follow-up is needed to determine how CD4 counts recover beyond 1 year and the degree to which prophylaxis and vigilance for CD4 recovery are necessary. In our institution we currently provide PJP prophylaxis and VZV reactivation prophylaxis for a minimum of 6 months or until recovery of CD4 count over 200 cells/ $\mu \mathrm{L}$, although there is physician variability in duration of prophylaxis and also with respect to the provision of IVIG to clinically asymptomatic patients. While we did not observe any PJP or Mycobacterium avium-intracellulare in our cohort, the level of CD4 suppression after CAR T-cell therapy may provide an impetus for prolonged or alternative prophylaxis in some patients. During the dates of this cohort study we typically revaccinated patients starting at 3 months post-CAR T-cell therapy in a manner analogous to our practice after autologous stem cell transplant. However, in this study we found that immunoglobulin levels nadir at 6 months and recover thereafter and we also noted recovery of B-cell aplasia and immunoglobulin levels while maintaining lymphoma remission, as is reported in other studies. . $^{1,0,11}$ This suggests that CD19 CAR T-cell therapy does not lead to a perma-
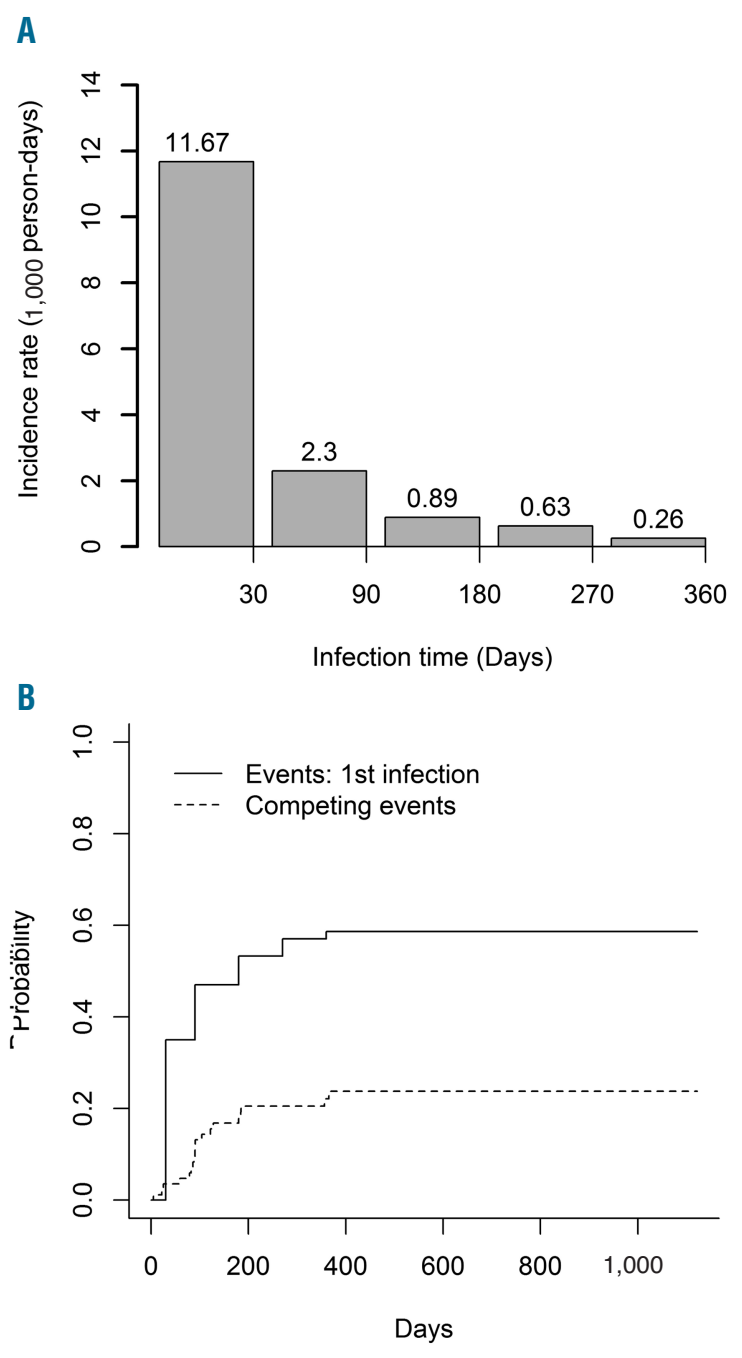

Figure 4. Infections occurring post-axi-cel. (A) Incidence rate of infection over time per 1,000 person-days, with time from axi-cel infusion on the X-axis. (B) Competing risk plot of the cumulative incidence of a patient's first infection. Death or progression were considered as competing events. axi-cel: axicabtagene ciloleucel.

nent loss of humoral immunity, which may account for a lower rate of opportunistic infections. Moreover, the available data presented here on HSV titers suggests that patients may not lose full protection against some infections. Similarly, Hill et al. ${ }^{18}$ recently reported on antiviral humoral immunity in 39 adults receiving CD19 CAR-T cell therapy and found that anti-measles IgG levels remained stable over time, possibly because long-lived plasma cells do not express CD19. ${ }^{18}$ Further study is required on the efficacy and optimal timing of infectious prophylaxis and vaccination after CAR T therapy.

Given the low rate of severe infections, this study is limited in that it cannot generate a risk prediction model for severe infection using baseline factors. A larger, ideally multicenter, cohort is needed to identify patients at risk of infection and to consider modifying treatment. Another issue is that we censored patients at the time of disease progression in order to reduce confounding introduced by subsequent therapy. However, cytopenias and poor immune reconstitution may interfere with therapies given for post-CAR $T$ relapse and further study is needed to 
identify if patients relapsing post-CAR $\mathrm{T}$ have unique infectious risks.

CAR T-cell therapy provides durable remissions in $40-50 \%$ of $\mathrm{R} / \mathrm{R}$ LBCL patients. As more patients receive this therapy and have prolonged remissions, we will need to understand and manage the unique survivorship issues that occur. Here we report that patients in remission after axi-cel have prolonged immunosuppression, with CD4 Tcell counts in particular remaining low for at least 1 year, and are at risk of infection in the short and long term.

\section{Disclosures}

$C B$ sits on the Advisory Board for Kite/Gilead; JCCh sit on the Advisory Board for Kite/Gilead, Novartis, Baver, Genetech and is a member of the Speaker Bureau for Genetech; BDS has is a consultant for Celgene/Juno, Adaptive, Kite/Gilead, Novartis, Pharmacyclics, Spectrum/Acroteca and AstraZeneca and has received research funding from Jazz and Incyte; JP-I is a consultant for Takeda, Abbvie, Janssen, Novartis, Gilead and Teva; MLD has received research funding from Celgene, Novartis, Atara and other financial support from Novartis, Precision Biosciences, Celyad, Bellicum, GlaxoSmithKline and holds stock options from Precision Biosciences, Adaptive Biotechnologies, Anixa Biosciences; FLL is a Consultant for Cellular Biomedicine Group, Inc. and a scientific advisor for Kite/Gilead, Novartis, and MDJ is consultant for Kite/Gilead and Novartis.

\section{Contributions}

$M D J$ and FLL designed and supervised the research; KML, $E Z, C A B, G S K, V L$ and $D N$ collected and analyzed data; GG $B C$ and $J K$ provided statistical analysis; JCC, $A B, F K, A L, T N$, $H D L, J P, B D S, R F, A E C, M L D$ and $B R D$ provided patient information and data.

All authors contributed to the writing of the manuscript.

\section{Funding}

This work was supported by NCI P30 CA076292 and NCI K23-CA201594.

\section{References}

1. Locke FL, Ghobadi A, Jacobson CA, et al. Long-term safety and activity of axicabtagene ciloleucel in refractory large B-cell lymphoma (ZUMA-1): a single-arm, multicentre, phase 1-2 trial. Lancet Oncol. 2019; 20(1):31-42.

2. Neelapu SS, Locke FL, Bartlett NL, et al. Axicabtagene ciloleucel CAR T-cell therapy in refractory large B-cell lymphoma. N Engl J Med. 2017;377(26):2531-2544.

3. Hill JA, Li D, Hay KA, et al. Infectious complications of CD19-targeted chimeric antigen receptor-modified T-cell immunotherapy. Blood. 2018;131(1):121-130.

4. Jain MD, Davila ML. Concise review: emerging principles from the clinical application of chimeric antigen receptor $\mathrm{T}$ cell therapies for B cell malignancies. Stem Cells. 2018;36(1):36-44

5. Lee DW, Gardner R, Porter DL, et al. Current concepts in the diagnosis and management of cytokine release syndrome. Blood. 2014; 124(2):188-195

6. Neelapu SS, Tummala S, Kebriaei P, et al. Chimeric antigen receptor T-cell therapy assessment and management of toxicities. Nat Rev Clin Oncol. 2018;15(1):47-62.

7.Locke FL, Go WY, Neelapu SS. Development and use of the anti-CD19 chimeric antigen receptor T-cell therapy axicabtagene ciloleucel in large B-cell lymphoma: a review. JAMA Oncol. 2019:1-10.
8. Fried S, Avigdor A, Bielorai B, et al. Early and late hematologic toxicity following CD19 CAR-T cells. Bone Marrow Transplant. 2019;54(10):1643-1650.

9. Shalabi H, Shah NN, Fry TJ, Yates B, Delbrook C. Chimeric antigen receptor induced cytopenia differs from chemotherapy induced myelosuppression. Blood. 2017; 130(Suppl 1):S5048.

10. Kochenderfer JN, Somerville RPT, Lu T, et al Long-duration complete remissions of dif fuse large B cell lymphoma after anti-CD19 chimeric antigen receptor $\mathrm{T}$ cell therapy. Mol Ther. 2017;25(10):2245-2253.

11. Schuster SJ, Svoboda J, Chong EA, et al. Chimeric antigen receptor $T$ cells in refractory B-cell lymphomas. N Engl J Med. 2017;377(26):2545-2554.

12. Locke FL, Neelapu SS, Bartlett NL, et al. Phase 1 results of ZUMA-1: a multicenter study of KTE-C19 anti-CD19 CAR T cell therapy in refractory aggressive lymphoma. Mol Ther. 2017;25(1):285-295.

13. Joffe E, Ariela Arad N, Bairey O, et al Persistently low lymphocyte counts after FCR therapy for chronic lymphocytic leukemia are associated with longer overall survival. Hematol Oncol. 2018;36(1):128135.

14. Ysebaert L, Gross E, Kuhlein E, et al. Immune recovery after fludarabinecyclophosphamide-rituximab treatment in B-chronic lymphocytic leukemia: implication for maintenance immunotherapy. Leukemia. 2010;24(7):1310-1316.
15. Park JH, Romero FA, Taur Y, et al. Cytokine release syndrome grade as a predictive marker for infections in patients with relapsed or refractory B-cell acute lymphoblastic leukemia treated with chimeric antigen receptor $\mathrm{T}$ cells. Clin Infect Dis. 2018;67(4):533-540.

16. Annibali O, Hohaus S, Marchesi F, et al. The neutrophil/lymphocyte ratio $>/=3.5$ is a prognostic marker in diffuse large B-cell lymphoma: a retrospective analysis from the database of the Italian regional network 'Rete Ematologica del Lazio per i Linfomi' (RELLI). Leuk Lymphoma. 2019; 60(14): 3386-3394.

17. Porrata LF, Ristow K, Habermann T, et al Predicting survival for diffuse large B-cell lymphoma patients using baseline neutrophil/lymphocyte ratio. Am J Hematol. 2010;85(11):896-899.

18. Hill JA, Krantz EM, Hay KA, et al. Durable preservation of antiviral antibodies after CD19-directed chimeric antigen receptor $\mathrm{T}$ cell immunotherapy. Blood Adv. 2019;3 22):3590-3601.

19. Hashmi H, Bachmeier C, Chavez JC, et al Haemophagocytic lymphohistiocytosis has variable time to onset following CD19 chimeric antigen receptor $\mathrm{T}$ cell therapy. Br J Haematol. 2019;187(2):e35-e38.

20. Nonomura Y, Yasumoto M, Yoshimura R, et al. Relationship between bone marrow cellularity and apparent diffusion coefficient. Magn Reson Imaging. 2001;13(5):757-760. 